\title{
EFFICIENT LIE-POISSON INTEGRATOR FOR SECULAR SPIN DYNAMICS OF RIGID BODIES
}

\author{
SŁAWOMIR BREITER \\ Astronomical Observatory, Adam Mickiewicz University, Słoneczna 36, PL 60-286 Poznań, Poland; breiter@amu.edu.pl \\ DAVID NESVORNÝ \\ Southwest Research Institute, 1050 Walnut Street, Suite 400, Boulder, CO 80302; davidn@boulder.swri.edu \\ AND \\ DAVID VOKROUHLICKÝ \\ Institute of Astronomy, Charles University, V Holešovičkách 2, 18000 Prague 8, Czech Republic; \\ vokrouhl@cesnet.cz \\ Received 2005 April 12; accepted 2005 May 25
}

\begin{abstract}
A fast and efficient numerical integration algorithm is presented for the problem of the secular evolution of the spin axis. Under the assumption that a celestial body rotates around its maximum moment of inertia, the equations of motion are reduced to the Hamiltonian form with a Lie-Poisson bracket. The integration method is based on the splitting of the Hamiltonian function, and so it conserves the Lie-Poisson structure. Two alternative partitions of the Hamiltonian are investigated, and second-order leapfrog integrators are provided for both cases. Non-Hamiltonian torques can be incorporated into the integrators with a combination of Euler and Lie-Euler approximations. Numerical tests of the methods confirm their useful properties of short computation time and reliability on long integration intervals.
\end{abstract}

Key words: celestial mechanics — methods: numerical — solar system: general

\section{INTRODUCTION}

Motion of celestial bodies is conventionally split into translation of the center of mass and rotation about the proper spin axis (Whittaker 1944). Planetary dynamics offers a plethora of cases in which translation and rotation interact via resonant phenomena. This manifests mainly through the translation mode affecting the rotation mode in either (or both) of the following two cases:

1. Rotation about the spin axis resonates with the orbital motion, such as the case of spin-orbit coupling of close planetary satellites, Mercury, or secondary components of asteroid binaries.

2. Precession of the spin axis resonates with precession of the orbital plane, such as the case of a number of planetary satellites (including our Moon), some terrestrial planets, possibly Saturn, and many asteroids.

It has been long understood that the occurrence of these resonant states reflects in many cases the past evolution of the spin and/or orbit due to dissipative effects. An accurate modeling of these evolutionary paths is a very interesting goal in planetary science. However, such ambitious models often depend on a number of unknown parameters, related to nongravitational torques, and are sensitive to the chaotic nature of the orbital evolution of gravitationally interacting bodies. As a result, efficient numerical tools are often very useful for sampling a wide range of parameters within a reasonable amount of CPU time.

In this paper we develop an efficient integrator to tackle the problems in planetary dynamics such as those described in item 2. As far as the problems of major planets are concerned, the rotation history of Mercury and Venus is a type 1 problem (Peale 1969, 1974; Goldreich \& Peale 1970; Ward \& de Campli
1979), but on the other hand, Earth and Mars remain distinguished cases in which secular spin dynamics (type 2) applies. The basic framework for this case was established in the classical paper by Colombo (1966), but the complications for Earth and Mars arise as a result of the necessity of accurate representation of the orbital evolution and its influence on the spin history. Ward $(1973,1974,1979)$ was the first to discover largescale oscillations in Mars' obliquity responding to forcing torques due to orbital evolution, itself driven by planetary perturbations. This field was later mastered by Laskar and collaborators using steadily improved solutions of planetary motion (e.g., Laskar \& Robutel 1993). Additional difficulty in understanding planetary spin evolution is due to long-term effects of nongravitational torques, such as tides (Peale 1974; Ward 1975, 1980; Néron de Surgy \& Laskar 1997), core-mantle friction (Rochester 1976), and/or atmospheric torques (Dobrovolskis \& Ingersoll 1980; Dobrovolskis 1980; Correia \& Laskar 2001, 2003; Correia et al. 2003). Modern paleoclimate studies for both Earth and Mars rely on these complicated models (Laskar et al. 2002, 2004a, 2004b). In addition, Ward \& Hamilton (2004) and Hamilton \& Ward (2004) have recently introduced an attractive model for explaining Saturn's large obliquity as a result of a resonance torque due to Neptune-raised terms in Saturn's orbital motion, modulated over the long term by a dissipating planetesimal disk. And not only the solar system planets are the subject of such studies. The growing interest in testing newly discovered planetary systems for habitable climate conditions also calls for an efficient obliquity tracking tool (Atobe et al. 2004).

In the realm of minor bodies (natural and artificial satellites, asteroids, and comets), resonant interactions of the orbital plane precession with the spin axis are so widely studied that any attempt at a brief bibliographic guide is doomed to be unsatisfactory. Here we only briefly touch on asteroid applications 
stemming from the work of Skoglöv (1997, 1998). Wider publicity for this field was attained after Vokrouhlický et al. (2003) solved, using a combination of secular spin-orbit resonant locking and the long-term influence of thermal torques (Rubincam 2000; Vokrouhlický \& Čapek 2002), the puzzling alignment of spin axes for five Koronis family member asteroids (Slivan 2002). Many more asteroids, especially those with low-inclination orbits and in the inner part of the main belt, are expected to reside in similar spin-orbit resonances (Rubincam et al. 2002). Intermittent resonant states are also expected for near-Earth asteroids, such as in the case of 433 Eros (Vokrouhlický et al. 2005). Growing knowledge of spin states for small nearEarth (and main-belt) asteroids, which will likely undergo a spectacular leap after data from future space-born missions are available (e.g., Kaasalainen 2004), will soon allow statistical studies of obliquity distribution across a wide range of sizes. Understanding the long-term (secular) dynamics of asteroid spin axes will become a necessary prerequisite to interpreting these data.

Such is the motivation for a development of an efficient numerical integration tool that may help in further studies of the above-mentioned problems. There exists a general purpose LiePoisson integrator, developed by Touma \& Wisdom (1994a), that handles the complete translation-rotation problem, but it involves a costly "Keplerian drift" part that can be suppressed under a few simplifying assumptions. Assuming principal axis rotation and averaging the appropriate Newton-Euler equations over orbital periods, we achieve a considerable gain in the computational efficiency. Postponing individual planetary applications to a forthcoming work, we aim at presenting numerical tools developed and optimized for this particular task.

In $\S 2$ we review the mathematical formulations of the secular spin dynamics for a rigid body in orbit about a force center and bring the underlying equations into a Hamiltonian form that allows application of Lie-Poisson splitting methods. In $\S 3$ we construct two alternative Lie-Poisson integrators based on different Hamiltonian splittings. The elementary maps can be used as building blocks for a high-order integrator, but we focus on the second-order "leapfrog" algorithm as the most convenient one. In $\S 4$ we extend our previous formulation, accounting for gravitational torques only, to the case in which arbitrary (dissipative) torques are required. In $\S 5$ we test the properties of the second-order leapfrog implementation for the secular spin-axis evolution.

\section{LIE-POISSON EQUATIONS OF ROTATION}

\subsection{Equations of Motion in an Inertial Frame}

In order to study the long-term evolution of the spin axis, we begin with averaged Newton-Euler equations describing the evolution of the unitary spin direction vector $\boldsymbol{e}$ under the action of the mean solar torque. ${ }^{1}$ The torque is perpendicular to the unitary vector $\boldsymbol{N}_{p}$, the latter being normal to the orbital plane. Let us assume that the body rotates around the principal axis of the inertia tensor. In an arbitrary inertial reference frame, e.g., the ecliptic-equinox of some epoch, we have (Bertotti et al. 2003, p. 176)

$$
\frac{d \boldsymbol{e}}{d t}=\boldsymbol{e} \times \alpha\left(\boldsymbol{N}_{p} \cdot \boldsymbol{e}\right) \boldsymbol{N}_{p}
$$

\footnotetext{
${ }^{1}$ For definiteness, we speak about an asteroid in orbit about the Sun. However, the same approach applies to a planet, or a satellite in orbit about a planet, etc.
}

where

$$
\alpha(t)=\frac{3 \mu \Delta}{2 \omega_{r} a^{3} \eta^{3}}
$$

is a function of the heliocentric gravitation parameter $\mu$ (gravity constant times the total mass of the system), dynamical ellipticity $\Delta$ defined in terms of the principal moments of inertia $I_{1} \leq I_{2} \leq I_{3}$ as

$$
\Delta=\frac{I_{3}-(1 / 2)\left(I_{1}+I_{2}\right)}{I_{3}}
$$

mean rotation frequency $\omega_{r}$, and two time-dependent orbital elements: the semimajor axis $a$ and eccentricity $\bar{e}$ defined by $\eta=\left(1-\bar{e}^{2}\right)^{1 / 2}$.

The time dependence of $\alpha$ is the result of planetary perturbations in $a$ and $\eta$. The orientation of the unit vector $\boldsymbol{N}_{p}$, normal to the orbital plane, is also time-dependent as a result of perturbations in inclination $I$ and in the longitude of the ascending node $\Omega$. If the body possesses a close or a distant satellite, one can recalibrate the ellipticity $\Delta$ to include information about the average solar torque exerted on the satellite orbit similarly to Néron de Surgy \& Laskar (1997) or Ward \& Hamilton (2004).

Equation (1) can be reformulated in the Lie-Poisson formalism to reveal its Hamiltonian, although noncanonical, structure (Olver 1993; Touma \& Wisdom 1994a). Let us introduce two operators: $\mathbf{Q}: \mathcal{R}^{3} \rightarrow \operatorname{so}(3)$ and $\boldsymbol{q}: \operatorname{so}(3) \rightarrow \mathcal{R}^{3}$ acting between the $\mathcal{R}^{3}$ Euclidian space and the so(3) Lie algebra of skewsymmetric matrices. For any vector $\boldsymbol{v} \in \mathcal{R}^{3}, \mathbf{Q}(\boldsymbol{v})$ is a skewsymmetric matrix:

$$
\mathbf{Q}(\boldsymbol{v})=\left(\begin{array}{ccc}
0 & -v_{3} & v_{2} \\
v_{3} & 0 & -v_{1} \\
-v_{2} & v_{1} & 0
\end{array}\right) .
$$

On the other hand, given a skew-symmetric matrix $\mathbf{V}=$ $[V]_{i j} \in$ so(3), we define

$$
\boldsymbol{q}(\mathbf{V})=\left(V_{32}, V_{13}, V_{21}\right)^{T} .
$$

These operators link the vector product and the matrix product. Given any pair of vectors $\boldsymbol{v}, \boldsymbol{w}$,

$$
v \times w=\mathbf{Q}(v) w
$$

and for any skew-symmetric matrix $\mathbf{V}$,

$$
\mathbf{V w}=q(\mathbf{V}) \times w .
$$

Equation (1) can be rewritten as

$$
\frac{d \boldsymbol{e}}{d t}=\mathbf{Q}(\boldsymbol{e})\left(\frac{\partial \mathcal{H}}{\partial \boldsymbol{e}}\right)^{T},
$$

where

$$
\mathcal{H}(\boldsymbol{e}, t)=\frac{\alpha}{2}\left(\boldsymbol{N}_{p} \cdot \boldsymbol{e}\right)^{2}
$$

and we use the convention of Battin (1987), i.e., for $f \in \mathcal{R}$, the derivative $\partial f / \partial \boldsymbol{e}$ is a row vector $\left(\partial f / \partial e_{1}, \partial f / \partial e_{2}, \partial f / \partial e_{3}\right)$, dual to the usual column vector. 
Let us now introduce a bilinear, skew-symmetric differential operator

$$
\{f ; g\}=\frac{\partial f}{\partial \boldsymbol{e}} \mathbf{Q}(\boldsymbol{e})\left(\frac{\partial g}{\partial \boldsymbol{e}}\right)^{T}
$$

Checking that $\{\ldots ; \ldots\}$ satisfies the Jacobi identity

$$
\{\{f ; g\} ; h\}+\{\{g ; h\} ; f\}+\{\{h ; f\} ; g\}=0
$$

and observing the linearity of $Q_{i j}$ with respect to the components of $\boldsymbol{e}$, we identify equation (10) as the definition of a LiePoisson bracket (Olver 1993). Accordingly, the equation of motion (8) is Hamiltonian (but not canonical),

$$
\frac{d \boldsymbol{e}}{d t}=\{\boldsymbol{e} ; \mathcal{H}\}
$$

with the Hamiltonian function $\mathcal{H}$. Note that for any scalar function $g$,

$$
\{\boldsymbol{e} \cdot \boldsymbol{e} ; g\}=0
$$

hence, all solutions of equation (12), regardless of the actual form of the Hamiltonian function, belong to the $\mathrm{SO}(3)$ group of rotations, respecting the $e_{1}^{2}+e_{2}^{2}+e_{3}^{2}=1$ constraint.

In the ecliptic-equinox inertial frame, $\boldsymbol{N}_{p}$ can be parameterized by means of the orbital inclination $I$ and longitude of the ascending node $\Omega$. The transformation of any vector $\boldsymbol{r}$ from the inertial frame to the nodal-orbital frame, where it becomes $\boldsymbol{r}^{\prime}=\mathbf{M} \boldsymbol{r}$, is defined by the rotation matrix

$$
\mathbf{M}=\mathbf{R}_{1}(I) \mathbf{R}_{3}(\Omega)=\left(\begin{array}{ccc}
\cos \Omega & \sin \Omega & 0 \\
-\cos I \sin \Omega & \cos I \cos \Omega & \sin I \\
\sin I \sin \Omega & -\sin I \cos \Omega & \cos I
\end{array}\right) .
$$

Because $N_{p}^{\prime}=(0,0,1)^{T}$, we find

$$
\boldsymbol{N}_{p}=\mathbf{M}^{T} \boldsymbol{N}_{p}^{\prime}=\left(\begin{array}{c}
\sin I \sin \Omega \\
-\sin I \cos \Omega \\
\cos I
\end{array}\right)=\left(\begin{array}{c}
2 p \sqrt{1-q^{2}-p^{2}} \\
-2 q \sqrt{1-q^{2}-p^{2}} \\
1-2\left(q^{2}+p^{2}\right)
\end{array}\right)
$$

In the latter form, we used nonsingular elements

$$
q+i p=\sin (I / 2) \exp i \Omega
$$

Thus, we can write the Hamiltonian explicitly as

$$
\mathcal{H}(\boldsymbol{e}, t)=\frac{\alpha}{2}\left[\left(e_{1} \sin \Omega-e_{2} \cos \Omega\right) \sin I+e_{3} \cos I\right]^{2}
$$

In the absence of planetary perturbations, $\alpha, \Omega$, and $I$ are constant, and we obtain the integral of motion $\mathcal{H}=$ const.

\subsection{Transformation of the Reference Frame}

The Hamiltonian (17) can be simplified by an appropriate rotation of the reference frame. But will an orthogonal (and possibly time-dependent) transformation of variables conserve the Hamiltonian structure of equation (12)? More precisely, there are two questions to be answered: Is the Lie-Poisson bracket invariant with respect to orthogonal transformations, and what kind of remainder should be added to the Hamiltonian if the transformation is time-dependent?

Let $\boldsymbol{e}^{\prime}=\mathbf{P}(t) \boldsymbol{e}$, where $\mathbf{P} \in \mathrm{SO}(3)$ is an arbitrary rotation matrix. For any pair of scalar functions $f, g$, their Lie-Poisson bracket (eq. [10]) transforms as

$$
\begin{aligned}
\{f ; g\} & =\frac{\partial f}{\partial \boldsymbol{e}} \mathbf{Q}(\boldsymbol{e})\left(\frac{\partial g}{\partial \boldsymbol{e}}\right)^{T} \\
& =\frac{\partial f}{\partial \boldsymbol{e}^{\prime}} \frac{\partial \boldsymbol{e}^{\prime}}{\partial \boldsymbol{e}}\left[\mathbf{P}^{T} \boldsymbol{e}^{\prime} \times\left(\frac{\partial g}{\partial \boldsymbol{e}^{\prime}} \frac{\partial \boldsymbol{e}^{\prime}}{\partial \boldsymbol{e}}\right)^{T}\right] \\
& =\frac{\partial f}{\partial \boldsymbol{e}^{\prime}} \mathbf{P}\left[\mathbf{P}^{T} \boldsymbol{e}^{\prime} \times\left(\frac{\partial g}{\partial \boldsymbol{e}^{\prime}} \mathbf{P}\right)^{T}\right] \\
& =\frac{\partial f}{\partial \boldsymbol{e}^{\prime}} \mathbf{P P}^{T} \operatorname{det} \mathbf{P}\left[\boldsymbol{e}^{\prime} \times\left(\frac{\partial g}{\partial \boldsymbol{e}^{\prime}}\right)^{T}\right] \\
& =\frac{\partial f}{\partial \boldsymbol{e}^{\prime}} \mathbf{Q}\left(\boldsymbol{e}^{\prime}\right)\left(\frac{\partial g}{\partial \boldsymbol{e}^{\prime}}\right)^{T} .
\end{aligned}
$$

Thus, we have demonstrated that the Lie-Poisson bracket (eq. [10]) is invariant with respect to rotations, and hence we can substitute $\boldsymbol{e}=\mathbf{P}^{T}(t) \boldsymbol{e}^{\prime}$ into equation (12), obtaining

$$
\frac{d\left(\mathbf{P}^{T} \boldsymbol{e}^{\prime}\right)}{d t}=\left\{\mathbf{P}^{T} \boldsymbol{e}^{\prime} ; \mathcal{H}\right\}
$$

where the Lie-Poisson bracket is evaluated with respect to $\boldsymbol{e}^{\prime}$ as in equation (18). After elementary transformations,

$$
\frac{d \boldsymbol{e}^{\prime}}{d t}=\left\{\boldsymbol{e}^{\prime} ; \mathcal{H}\right\}-\mathbf{P} \dot{\mathbf{P}}^{T} \boldsymbol{e}^{\prime}
$$

From the properties of rotation matrices, we know that $\mathbf{P} \dot{\mathbf{P}}^{T}$ is a skew-symmetric matrix, and

$$
\boldsymbol{q}\left(\mathbf{P} \dot{\mathbf{P}}^{T}\right)=\boldsymbol{\omega}
$$

where $\boldsymbol{\omega}$ is the angular rate vector of the rotating reference frame. This allows us to rewrite the equations of motion as

$$
\frac{d \boldsymbol{e}^{\prime}}{d t}=\left\{\boldsymbol{e}^{\prime} ; \mathcal{H}\right\}+\boldsymbol{e}^{\prime} \times \boldsymbol{\omega}
$$

But

$$
\boldsymbol{e}^{\prime} \times \boldsymbol{\omega}=\mathbf{Q}\left(\boldsymbol{e}^{\prime}\right) \boldsymbol{\omega}=\left\{\boldsymbol{e}^{\prime} ; \boldsymbol{\omega} \cdot \boldsymbol{e}^{\prime}\right\}
$$

hence, from the linearity of Lie-Poisson brackets we obtain the Hamiltonian equations of motion in a rotating frame:

$$
\frac{d \boldsymbol{e}^{\prime}}{d t}=\left\{\boldsymbol{e}^{\prime} ; \mathcal{H}^{\prime}\right\}
$$

where

$$
\mathcal{H}^{\prime}\left(\boldsymbol{e}^{\prime}, t\right)=\mathcal{H}\left(\mathbf{P}^{T} \boldsymbol{e}^{\prime}, t\right)+\boldsymbol{\omega} \cdot \boldsymbol{e}^{\prime}
$$




\subsection{Asteroid Spin Axis in an Orbital Frame}

Let us adopt the orbital reference frame defined by orthonormal vectors $\boldsymbol{i}^{\prime}, \boldsymbol{j}^{\prime}, \boldsymbol{k}^{\prime}$, where $\boldsymbol{k}^{\prime}$ is normal to the osculating orbital plane. Then, the Hamiltonian function (eq. [25]), where $\mathcal{H}$ is given by equation (9), takes the form

$$
\mathcal{H}^{\prime}=\frac{\alpha(t)}{2}\left(e_{3}^{\prime}\right)^{2}+\omega_{1}(t) e_{1}^{\prime}+\omega_{2}(t) e_{2}^{\prime}+\omega_{3}(t) e_{3}^{\prime} .
$$

Directing $\boldsymbol{i}^{\prime}$ toward the ascending node of the orbit may sometimes be preferred, but usually this is not the best choice. It is much more convenient to rotate $\boldsymbol{i}^{\prime}$ backward by an angle $-\Omega$ along the orbital plane. The gain is twofold: not only do we avoid problems of undefined nodes when $\sin I=0$, but we also partially compensate for the reference frame motion due to $\dot{\Omega}$ when the inclination is small.

Let us use the symbol $\boldsymbol{v}$ to stand for the spin vector $\boldsymbol{e}^{\prime}$ in this particular reference frame. Its components will be $\boldsymbol{v}=(x, y, z)^{T}$. Hence, the transformation to the new reference frame is $\boldsymbol{v}=\mathbf{P} \boldsymbol{e}$, where

$$
\mathbf{P}=\mathbf{R}_{3}(-\Omega) \mathbf{R}_{1}(I) \mathbf{R}_{3}(\Omega),
$$

and, accordingly,

$$
\boldsymbol{\omega}=\boldsymbol{q}\left(\mathbf{P} \dot{\mathbf{P}}^{T}\right)=\left(\begin{array}{c}
\dot{I} \cos \Omega-\dot{\Omega} \sin I \sin \Omega \\
\dot{I} \sin \Omega+\dot{\Omega} \sin I \cos \Omega \\
-(1-\cos I) \dot{\Omega}
\end{array}\right)
$$

The location of the spin axis in the reference frame $\boldsymbol{i}^{\prime}, \boldsymbol{j}^{\prime}, \boldsymbol{k}^{\prime}$ can be traditionally expressed in terms of two angles: colatitude $\varepsilon$, called the obliquity, and longitude $\lambda$, so that

$$
\boldsymbol{v}=\left(\begin{array}{l}
x \\
y \\
z
\end{array}\right)=\left(\begin{array}{c}
\sin \varepsilon \cos \lambda \\
\sin \varepsilon \sin \lambda \\
\cos \varepsilon
\end{array}\right)
$$

In order to be in line with a long tradition (e.g., Newcomb 1895), we can alternatively use an angle

$$
\psi=\frac{\pi}{2}-\lambda=\frac{\pi}{2}-\arctan \frac{y}{x}=\arctan \frac{x}{y},
$$

i.e.,

$$
\boldsymbol{v}=\left(\begin{array}{c}
\sqrt{1-z^{2}} \sin \psi \\
\sqrt{1-z^{2}} \cos \psi \\
z
\end{array}\right)
$$

Since the paper of Laskar (1986), the components of $\boldsymbol{\omega}$ are often replaced by closely related quantities $\mathcal{A}, \mathcal{B}$, and $\mathcal{C}$, defined as

$$
\begin{gathered}
\mathcal{A}(t)=\omega_{1}=\frac{2(\dot{q}+p \mathcal{C})}{\sqrt{1-p^{2}-q^{2}}}, \\
\mathcal{B}(t)=\omega_{2}=\frac{2(\dot{p}-q \mathcal{C})}{\sqrt{1-p^{2}-q^{2}}}, \\
\mathcal{C}(t)=-\frac{\omega_{3}}{2}=q \dot{p}-p \dot{q},
\end{gathered}
$$

where $p$ and $q$ were defined in equation (16). Using our ultimate symbols, we rewrite the Hamiltonian (26) as

$$
\mathcal{H}^{\prime}(\boldsymbol{v}, t)=\frac{\alpha(t)}{2} z^{2}+\mathcal{A}(t) x+\mathcal{B}(t) y-2 \mathcal{C}(t) z
$$

the Lie-Poisson bracket as

$$
\{f ; g\}=\frac{\partial f}{\partial \boldsymbol{v}} \mathbf{Q}(\boldsymbol{v})\left(\frac{\partial g}{\partial \boldsymbol{v}}\right)^{T}
$$

and the equations of motion as

$$
\dot{\boldsymbol{v}}=\left\{\boldsymbol{v} ; \mathcal{H}^{\prime}\right\}
$$

\subsection{Reduction to the Canonical Form}

Our equations of motion are locally equivalent to the canonical form given by Laskar \& Robutel (1993). Let us exclude the poles $z= \pm 1$ and introduce two variables on the surface of the sphere $v^{2}=1$ : the precession angle $\psi$ from equation (30) and some unspecified function of colatitude $X(\varepsilon)$. Under the new parameterization $\boldsymbol{h}=(\psi, X)^{T}$, the Lie-Poisson bracket $\{f ; g\}$ is transformed into a reduced operator $(f ; g)$, according to the transformation rule (Olver 1993)

$$
(f ; g)=\left(\frac{\partial f}{\partial \boldsymbol{h}}\right)\left(\begin{array}{ll}
\{\psi ; \psi\} & \{\psi ; X\} \\
\{X ; \psi\} & \{X ; X\}
\end{array}\right)\left(\frac{\partial g}{\partial \boldsymbol{h}}\right)^{T}
$$

i.e.,

$$
(f ; g)=\frac{\partial X}{\partial z}\left(\frac{\partial f}{\partial \psi} \frac{\partial g}{\partial X}-\frac{\partial f}{\partial X} \frac{\partial g}{\partial \psi}\right) .
$$

Thus, an obvious choice $X=z=\cos \varepsilon$ brings in the classical, canonically conjugate pair $\psi, X$ and the canonical Poisson bracket $(\psi ; X)=1$. Using our Hamiltonian (33) expressed in terms of canonical variables,

$$
\mathcal{H}^{\prime}=\frac{\alpha(t)}{2} X^{2}-2 \mathcal{C}(t) X+\sqrt{1-X^{2}}[\mathcal{A}(t) \sin \psi+\mathcal{B}(t) \cos \psi]
$$

we obtain the canonical equations of Laskar \& Robutel (1993),

$$
\begin{aligned}
\dot{X}=\left(X ; \mathcal{H}^{\prime}\right) & =-\frac{\partial \mathcal{H}^{\prime}}{\partial \psi}=\sqrt{1-X^{2}}(-\mathcal{A} \cos \psi+\mathcal{B} \sin \psi) \\
\dot{\psi}=\left(\psi ; \mathcal{H}^{\prime}\right) & =\frac{\partial \mathcal{H}^{\prime}}{\partial X} \\
& =\alpha X-2 \mathcal{C}-\frac{X}{\sqrt{1-X^{2}}}(\mathcal{A} \sin \psi+\mathcal{B} \cos \psi)
\end{aligned}
$$

The canonical equations of motion are only two, as compared to three components of $\dot{v}$, but they possess serious drawbacks: singularities at $X^{2}=1$ and a more complicated form.

\subsection{Extended Phase Space}

Introducing an additional canonically conjugate pair of variables $u, E$, we can replace the time-dependent Hamiltonian 
$\mathcal{H}^{\prime}(\boldsymbol{v}, t)$ from equation (33) with a new, conservative function $\mathcal{K}$ of five variables $\boldsymbol{w}=(x, y, z, E, u)$,

$$
\begin{aligned}
\mathcal{K}(\boldsymbol{w}) & =\mathcal{H}^{\prime}(\boldsymbol{v}, u)+E \\
& =\frac{\alpha(u)}{2} z^{2}+\mathcal{A}(u) x+\mathcal{B}(u) y-2 \mathcal{C}(u) z+E=\mathrm{const},
\end{aligned}
$$

where $\alpha, \mathcal{A}, \mathcal{B}$, and $\mathcal{C}$ become functions of the formal, timelike $u$. In the extended phase space, the Lie-Poisson bracket (34) can be replaced by

$$
\{f ; g\}=\frac{\partial f}{\partial \boldsymbol{w}} \mathbf{J}(\boldsymbol{v})\left(\frac{\partial g}{\partial \boldsymbol{w}}\right)^{T}
$$

where the structure matrix $\mathbf{J}$ is

$$
\mathbf{J}(\boldsymbol{v})=\left(\begin{array}{ccccc}
0 & -z & y & 0 & 0 \\
z & 0 & -x & 0 & 0 \\
-y & x & 0 & 0 & 0 \\
0 & 0 & 0 & 0 & -1 \\
0 & 0 & 0 & 1 & 0
\end{array}\right)
$$

The equations of motion in the extended phase space are $\dot{\boldsymbol{w}}=\{\boldsymbol{w} ; \mathcal{K}\}$ or, explicitly,

$$
\begin{gathered}
\dot{x}=\alpha(u) z y-2 \mathcal{C}(u) y-\mathcal{B}(u) z, \\
\dot{y}=-\alpha(u) z x+2 \mathcal{C}(u) x+\mathcal{A}(u) z, \\
\dot{z}=-\mathcal{A}(u) y+\mathcal{B}(u) x, \\
\dot{E}=-\frac{z^{2}}{2} \frac{d \alpha}{d u}-x \frac{d \mathcal{A}}{d u}-y \frac{d \mathcal{B}}{d u}+2 z \frac{d \mathcal{C}}{d u}, \\
\dot{u}=1 .
\end{gathered}
$$

These last equations deserve a few comments. First we note that $t$ and $u$ differ only by an arbitrary additive constant, with $u=t$ as the most natural choice. The evolution of the energy-like variable $E$ counterbalances the varying $\mathcal{H}^{\prime}$ in order to provide the constant $\mathcal{K}$. As a matter of fact, one can solve the equations for $\boldsymbol{v}$ without any knowledge of the values of $E$. Thus, we do not pay attention to the evolution of this "variable in being."

\section{EXPLICIT LIE-POISSON INTEGRATORS}

According to the principles of the so-called splitting method (McLachlan \& Quispel 2002), an explicit integrator can be built as a composition of solutions to the equations of motion generated by separate terms of a partitioned Hamiltonian. We consider two ways of splitting $\mathcal{K}$ that differ only by the placement of the $E$-variable. Surprisingly, the two resulting algorithms are completely different.

\subsection{Three-Term Splitting Method (LP3)}

The first integration algorithm results if we identify three different, noncommuting terms of the Hamiltonian:

$$
\mathcal{K}(\boldsymbol{w})=\mathcal{K}_{0}(E)+\mathcal{K}_{1}(z, u)+\mathcal{K}_{2}(\boldsymbol{v}, u),
$$

where

$$
\begin{gathered}
\mathcal{K}_{0}=E, \\
\mathcal{K}_{1}=\frac{\alpha(u)}{2} z^{2}, \\
\mathcal{K}_{2}=\mathcal{A}(u) x+\mathcal{B}(u) y-2 \mathcal{C}(u) z .
\end{gathered}
$$

Each $\mathcal{K}_{j}$ generates the Lie-Poisson equations of motion

$$
\dot{\boldsymbol{w}}=\left\{\boldsymbol{w} ; \mathcal{K}_{j}\right\} \equiv \mathcal{L}_{j} \boldsymbol{w}
$$

which can be solved explicitly. The solutions can be formally represented by means of the operators $\Psi_{j, \tau}=\exp \left(\tau \mathcal{L}_{j}\right)$, which map initial conditions $\boldsymbol{w}(t)$ onto

$$
\boldsymbol{w}(t+\tau)=\Psi_{j, \tau} \boldsymbol{w}(t)=\exp \left(\tau \mathcal{L}_{j}\right) \boldsymbol{w}(t)
$$

The flow generated by $\mathcal{K}_{0}$ is quite trivial. The equations of motion $\dot{\boldsymbol{w}}=\mathcal{L}_{0} \boldsymbol{w}$, or

$$
\dot{\boldsymbol{v}}=0, \quad \dot{E}=0, \quad \dot{u}=1,
$$

amount to a simple propagation of the formal time $u$ :

$$
\Psi_{0, \tau} \boldsymbol{v}=\boldsymbol{v}, \quad \Psi_{0, \tau} u=u+\tau, \quad \Psi_{0, \tau} E=E
$$

Note that the remaining terms $\mathcal{K}_{1}$ and $\mathcal{K}_{2}$ do not contain $E$; hence, the value of $u$ is fixed in the next two flows.

The $\mathcal{K}_{1}$ term consists of a monomial $z^{2}$ times a constant. Accordingly, the equations of motion $\dot{\boldsymbol{w}}=\mathcal{L}_{1} \boldsymbol{w}$ should describe rotation around the $z$-axis with the rate depending on $\alpha z$, which is indeed the case. The physics behind

$$
\begin{gathered}
\dot{x}=\alpha(u) z y, \quad \dot{y}=-\alpha(u) z x, \quad \dot{z}=0, \\
\dot{E}=-\frac{d \alpha(u)}{d u} \frac{z^{2}}{2}, \quad \dot{u}=0
\end{gathered}
$$

is clear: a regular precession of the spin axis forced by the mean solar torque that vanishes if $\epsilon=\frac{1}{2} \pi$ (i.e., $z=0$ ). Solving equations (51), we obtain a nonlinear map for $\boldsymbol{v}$,

$$
\Psi_{1, \tau} \boldsymbol{v}=\left(\begin{array}{ccc}
\cos \varphi_{1} & \sin \varphi_{1} & 0 \\
-\sin \varphi_{1} & \cos \varphi_{1} & 0 \\
0 & 0 & 1
\end{array}\right) \boldsymbol{v}
$$

with $\varphi_{1}=\alpha(u) z \tau$ and

$$
\Psi_{1, \tau} u=u
$$

Knowledge of $\Psi_{1, \tau} E$ is not required.

The equations of motion $\dot{\boldsymbol{w}}=\mathcal{L}_{2} \boldsymbol{w}$, generated by the last term $\mathcal{K}_{2}$, are linear. For $\boldsymbol{v}$ they represent the effect of inertial forces due to the planetary perturbations of the reference frame. Recall that $u$ is fixed, so the reference frame rotates with a constant rate. Thus,

$$
\dot{\boldsymbol{v}}=\left(\begin{array}{ccc}
0 & -2 \mathcal{C}(u) & -\mathcal{B}(u) \\
2 \mathcal{C}(u) & 0 & \mathcal{A}(u) \\
\mathcal{B}(u) & -\mathcal{A}(u) & 0
\end{array}\right) \boldsymbol{v}=-\boldsymbol{\omega} \times \boldsymbol{v} .
$$

The solution of equation (54) is

$$
\Psi_{2, \tau} \boldsymbol{v}=\omega^{-2} \mathbf{M} \boldsymbol{v}
$$


with the following elements of matrix $\mathbf{M}$ :

$$
\begin{gathered}
M_{11}=\mathcal{A}^{2}+\left(\mathcal{B}^{2}+4 \mathcal{C}^{2}\right) \cos (\omega \tau), \\
M_{12}=2 \mathcal{A B} \sin ^{2} \frac{\omega \tau}{2}-2 \mathcal{C} \omega \sin (\omega \tau), \\
M_{13}=-4 \mathcal{A C} \sin ^{2} \frac{\omega \tau}{2}-\mathcal{B} \omega \sin (\omega \tau), \\
M_{21}=2 \mathcal{A B} \sin ^{2} \frac{\omega \tau}{2}+2 \mathcal{C} \omega \sin (\omega \tau), \\
M_{22}=\mathcal{B}^{2}+\left(\mathcal{A}^{2}+4 \mathcal{C}^{2}\right) \cos (\omega \tau), \\
M_{23}=-4 \mathcal{B C} \sin ^{2} \frac{\omega \tau}{2}+\mathcal{A} \omega \sin (\omega \tau), \\
M_{31}=-4 \mathcal{A C} \sin ^{2} \frac{\omega \tau}{2}+\mathcal{B} \omega \sin (\omega \tau), \\
M_{32}=-4 \mathcal{B C} \sin ^{2} \frac{\omega \tau}{2}-\mathcal{A} \omega \sin (\omega \tau), \\
M_{33}=4 \mathcal{C}^{2}+\left(\mathcal{A}^{2}+\mathcal{B}^{2}\right) \cos (\omega \tau),
\end{gathered}
$$

and

$$
\omega=\sqrt{\mathcal{A}^{2}+\mathcal{B}^{2}+4 \mathcal{C}^{2}} .
$$

For the remaining two variables,

$$
\dot{E}=-\boldsymbol{v} \cdot \frac{d \omega}{d u}, \quad \dot{u}=0,
$$

so

$$
\Psi_{2, \tau} u=u
$$

and, as usual, we pay no attention to the value of $E$.

Knowing the explicit form of each $\mathcal{L}_{j}$-generated flow, we can approximate the solution of equations (42) for step-size interval $h$, composing the $\Psi_{j, \beta_{k} h}$ maps with appropriate step subdivision constants $\beta_{k}$. The basic, first-order approximation is obtained as

$$
\boldsymbol{w}(t+h)=\Psi_{2, h} \circ \Psi_{1, h} \circ \Psi_{0, h} \boldsymbol{w}(t)+O\left(h^{2}\right) .
$$

Throughout the paper, any composition of $\Psi_{0}, \Psi_{1}$, and $\Psi_{2}$ maps is referred to as an LP3 integrator.

\subsection{Two-Term Splitting Method (LP2)}

Intuitively, one might think that freezing the rates of the reference frame motion, effected by isolating $E$ from the remaining terms of $\mathcal{K}$, should produce the simplest algorithm. This is not so, however. Let us join $\mathcal{K}_{0}$ and $\mathcal{K}_{2}$ into a single term $\mathcal{K}_{20}$, so that we have

$$
\mathcal{K}=\mathcal{K}_{1}+\mathcal{K}_{20}
$$

where

$$
\begin{gathered}
\mathcal{K}_{1}=\frac{\alpha(u)}{2} z^{2}, \\
\mathcal{K}_{20}=\mathcal{A}(u) x+\mathcal{B}(u) y-2 \mathcal{C}(u) z+E=\boldsymbol{\omega}(u) \cdot \boldsymbol{v}+E .
\end{gathered}
$$

There is no need to repeat the discussion of $\dot{\boldsymbol{w}}=\mathcal{L}_{1} \boldsymbol{w}$, because the operator $\Psi_{1, \tau}$ has already been presented in equations (52) and (53). The second term leads to $\dot{\boldsymbol{w}}=\mathcal{L}_{20} \boldsymbol{w}$, that is

$$
\begin{gathered}
\dot{\boldsymbol{v}}=\boldsymbol{v} \times \boldsymbol{\omega}, \\
\dot{E}=-\boldsymbol{v} \cdot \frac{d \boldsymbol{\omega}}{d u}=-\boldsymbol{v} \cdot \dot{\boldsymbol{\omega}} \\
\dot{u}=1 .
\end{gathered}
$$

Equations (54) and (64) or (58) and (65) may look similar at first glimpse, but it is $\dot{u}=1$ that makes the difference and leads to $d \boldsymbol{\omega} / d u=\dot{\omega}$. The best way to solve equation (64) is to think about its physics. It describes the vector $\boldsymbol{e}$, constant in the inertial frame, as seen in the noninertial reference frame $\boldsymbol{i}^{\prime}, \boldsymbol{j}^{\prime}, \boldsymbol{k}^{\prime}$, where it becomes $\boldsymbol{e}^{\prime}=v$. In other words,

$$
\Psi_{20, \tau} \boldsymbol{v}=\mathbf{M}_{20} \boldsymbol{v},
$$

where $\mathbf{M}_{20}$ is the matrix of rotation to the inertial frame at the initial $t=u$ and then back to the orbital frame at $u+\tau$. In terms of $\Omega_{0}=\Omega(u), \Omega_{1}=\Omega(u+\tau), I_{0}=I(u)$, and $I_{1}=$ $I(u+\tau)$,

$$
\mathbf{M}_{20}=\mathbf{R}_{3}\left(-\Omega_{1}\right) \mathbf{R}_{1}\left(I_{1}\right) \mathbf{R}_{3}\left(\Omega_{1}-\Omega_{0}\right) \mathbf{R}_{1}\left(-I_{0}\right) \mathbf{R}_{3}\left(\Omega_{0}\right) .
$$

It is better, however, to use the nonsingular elements $q_{0}, p_{0}$ and $q_{1}, p_{1}$. If

$$
\mathbf{R}(q, p)=\left(\begin{array}{ccc}
1-2 p^{2} & 2 q p & 2 p \nu \\
2 q p & 1-2 q^{2} & -2 q \nu \\
-2 p \nu & 2 q \nu & 2 \nu^{2}-1
\end{array}\right),
$$

where $\nu=\left(1-q^{2}-p^{2}\right)^{1 / 2}$, then

$$
\mathbf{M}_{20}=\left[\mathbf{R}\left(q_{1}, p_{1}\right)\right]^{T} \mathbf{R}\left(q_{0}, p_{0}\right) .
$$

Ignoring the propagation of $E$, we stop at

$$
\Psi_{20, \tau} u=u+\tau
$$

which completes $\Psi_{20}$.

Therefore, we define an alternative first-order map,

$$
\boldsymbol{w}(t+h)=\Psi_{1, h} \circ \Psi_{20, h} \boldsymbol{w}(t)+O\left(h^{2}\right) .
$$

Similarly to the previous algorithm, we call all maps composed of $\Psi_{1}$ and $\Psi_{20}$ the LP2 integrators.

\subsection{Other Possibilities}

LP2 and LP3 integrators are, in our opinion, the most favorable ways of splitting the Hamiltonian $\mathcal{K}$. Obviously, a wide variety of different splitting strategies are possible, each having its own advantages and drawbacks. For example, a further partition of $\mathcal{K}_{2}$ in the LP3 method into three Hamiltonians $\mathcal{K}_{21}=$ $\mathcal{A}(u) x, \mathcal{K}_{22}=\mathcal{B}(u) y$, and $\mathcal{K}_{23}=-2 \mathcal{C}(u) z$ would result in replacing the rotation matrix $\mathbf{M}$ with a product of three elementary rotations around subsequent axes. Simplicity would be gained at the expense of additional error resulting from such an approximation. Moreover, this kind of decomposition of rotation is known to be sensitive to the choice of the rotation sequence. A 
similar partition within the LP2 scheme contradicts the main idea of the latter. Another potentially promising splitting can be imagined if the explicit Fourier series are known for the timedependent coefficients. Extracting the constant term from $\alpha$ and $\mathcal{C}$, and the first harmonic from $\mathcal{A}$ and $\mathcal{B}$, would define an integrable Hamiltonian describing the Colombo top (Colombo 1966; Henrard \& Murigande 1987). Then, in many cases, the remainder Hamiltonian would be small, resulting in the Wisdom-Holman type method. But an explicit solution of the Colombo top problem has not been published yet, and presumably it will have a complicated and time-consuming form involving elliptic functions and integrals.

\subsection{The Reasons for Leapfrogging}

The first-order integration algorithms presented in the previous sections have only one advantage: they use a uniform grid of the orbital plane osculating elements, separated by a fixed integration step $h$. But the low accuracy of first-order integrators is seldom satisfactory. On the other hand, higher order integrators use backward substeps that increase round-off errors (Sheng 1989), and they require a nonuniform spacing of the osculating elements. The former disadvantage probably explains the ubiquity of second-order leapfrog integrators, known also as the Störmer-Verlet method or even Newton-Störmer-Verlet (Hairer et al. 2003), according to Feynman (1967), who discovered this algorithm in Isaac Newton's Principia. The leapfrog uses only the forward, uniformly spaced substeps, which speaks in favor of this method in our case, although McLachlan \& Quispel (2002) provide a different second-order splitting method with a smaller estimate but using unevenly spaced substeps. There are also higher order splitting algorithms without backward substeps, found by Laskar \& Robutel (2001), but the methods are limited to the case in which the Hamiltonian is split into a single main term and a small perturbing part. As a matter of fact, this may be the case in our LP 2 method, when $z \alpha \ll \omega$ or vice versa. Finally, "symplectic correctors" can be applied to improve firstor second-order integrators (Wisdom et al. 1996; McLachlan 1996), but this device is worth the effort mostly in the small perturbation case. For these reasons, having pointed to other possibilities, we discuss only the Störmer-Verlet approach.

The leapfrog method applied to the Hamiltonian LP3 map results in

$$
\begin{gathered}
\boldsymbol{w}(t+h)=\Psi_{0, h / 2} \circ \Psi_{1, h / 2} \circ \Psi_{2, h} \circ \Psi_{1, h / 2} \\
\circ \Psi_{0, h / 2} \boldsymbol{w}(t)+O\left(h^{3}\right) .
\end{gathered}
$$

Similarly, the leapfrog extension of LP2 is

$$
\boldsymbol{w}(t+h)=\Psi_{1, h / 2} \circ \Psi_{20, h} \circ \Psi_{1, h / 2} \boldsymbol{w}(t)+O\left(h^{3}\right) .
$$

Let us observe that in the LP3 case defined by equation (73), osculating elements of the orbit and their derivatives are required at the epochs $t_{0}+(2 j+1)(h / 2)$, whereas, according to equation (74), the LP2 leapfrog needs the orbital ephemeris at $t_{0}+j h$. In any case, however, these are grids with the same density that would be required by a first-order method.

\section{NON-HAMILTONIAN TORQUES}

The algorithms presented in the previous sections have been designed to handle the evolution of the spin vector $v$ under the action of the averaged solar torque. The torque has been Hamiltonian and did not affect the length of the angular momentum vector
$\boldsymbol{L}=I_{3} \omega_{r} \boldsymbol{v}$; in other words, $L=I_{3} \omega_{r}$ was constant. In this section we present the general outline of recipes that allow us to incorporate an arbitrary, non-Hamiltonian torque $\boldsymbol{T}_{n}$,

$$
\boldsymbol{T}_{n}=I_{3} \omega_{r} \boldsymbol{T}
$$

into the integrator.

Let us generalize equation (35) as

$$
\dot{\boldsymbol{L}}=\left\{\boldsymbol{L}, \mathcal{H}^{\prime}\right\}+\boldsymbol{T}_{n}=\boldsymbol{L} \times\left(\frac{\partial \mathcal{H}^{\prime}}{\partial \boldsymbol{v}}\right)^{T}+\boldsymbol{T}_{n},
$$

where the Lie-Poisson bracket is defined as in equation (34). Touma \& Wisdom (1994b) proposed to handle $\boldsymbol{T}_{n}$ by adding to their Lie-Poisson maps the simplest Euler approximation:

$$
\boldsymbol{L}\left(t_{0}+h\right) \approx \boldsymbol{L}\left(t_{0}\right)+\boldsymbol{T}_{n}\left(t_{0}\right) h
$$

Simplicity is the only advantage of this approach. Actually, the Euler approximation is commonly used in geometric integration tutorials as a reference example whose only purpose is to demonstrate the superiority of anything else (Budd \& Piggott 2003; Hairer 2001). Its drawbacks are twofold: the appearance of a spurious trend in the length of $\boldsymbol{L}$ and an incorrect rate for the energy dissipation. Both these problems can be resolved when the functional form of the torque is specified. In general, the former problem can also be alleviated by the following procedure, which does not require explicit knowledge of $\boldsymbol{T}_{n}$. We decompose $\boldsymbol{T}_{n}$ into the part responsible for the change of $\omega_{r}$ and the remainder that generates rotation.

One can easily verify that the length $L$ is affected only by the component of $\boldsymbol{T}_{n}$ parallel to the spin vector $\boldsymbol{v}$. Indeed,

$$
\begin{aligned}
\frac{1}{2} \frac{d(\boldsymbol{L} \cdot \boldsymbol{L})}{d t} & =\dot{\boldsymbol{L}} \cdot \boldsymbol{L} \\
& =\boldsymbol{L} \cdot\left[\boldsymbol{L} \times\left(\frac{\partial \mathcal{H}^{\prime}}{\partial \boldsymbol{v}}\right)^{T}\right]+\boldsymbol{L} \cdot \boldsymbol{T}_{n} \\
& =\boldsymbol{L} \cdot \boldsymbol{T}_{n} .
\end{aligned}
$$

On the other hand, assuming a constant moment of inertia $\dot{I}_{3}=$ 0 , we have

$$
\frac{1}{2} \frac{d(\boldsymbol{L} \cdot \boldsymbol{L})}{d t}=I_{3}^{2} \omega_{r} \dot{\omega}_{r}
$$

Therefore, we obtain

$$
I_{3} \dot{\omega}_{r}=\boldsymbol{v} \cdot \boldsymbol{T}_{n}
$$

Simple vectorial identities lead to

$$
\boldsymbol{T}_{n}=-\boldsymbol{v} \times\left(\boldsymbol{v} \times \boldsymbol{T}_{n}\right)+\left(\boldsymbol{v} \cdot \boldsymbol{T}_{n}\right) \boldsymbol{v} .
$$

Thus, we can decompose equation (76) into

$$
\begin{gathered}
\dot{\omega}_{r}=\omega_{r} \boldsymbol{v} \cdot \boldsymbol{T}=T_{\|} \\
\dot{\boldsymbol{v}}=\left\{\boldsymbol{v}, \mathcal{H}^{\prime}\right\}+\boldsymbol{v} \times \boldsymbol{T}^{*}=\boldsymbol{v} \times\left[\left(\frac{\partial \mathcal{H}^{\prime}}{\partial \boldsymbol{v}}\right)^{T}+\boldsymbol{T}^{*}\right],
\end{gathered}
$$


where

$$
T^{*}=T \times v=-\mathbf{Q}(v) T .
$$

Passing to the extended phase space, we can generalize equations (42), obtaining

$$
\begin{gathered}
\dot{\omega}_{r}=T_{\|}, \quad \dot{\boldsymbol{v}}=\{\boldsymbol{v}, \mathcal{K}\}+\boldsymbol{v} \times \boldsymbol{T}^{*} \\
\dot{E}=-\frac{\partial \mathcal{K}}{\partial u}-\left(\frac{\partial \mathcal{K}}{\partial \boldsymbol{v}}\right) \mathbf{Q}(\boldsymbol{v}) \boldsymbol{T}^{*}-\frac{z^{2}}{2} \frac{\alpha(u)}{\omega_{r}} T_{\|}, \quad \dot{u}=1 .
\end{gathered}
$$

Note that $E$ remains defined as $E=-\mathcal{K}$, but as usual we ignore its actual value. Suppressing the assumption of constant $I_{3}$, which can be unrealistic for liquid planets, one should replace $T_{\|}$in equations (85) with $T_{\|}-\omega_{r} I_{3}^{-1} \dot{I}_{3}$, but we do not consider this case in the present study.

Following the "splitting method" recipe, we can maintain the LP2 or LP3 maps for the Hamiltonian evolution of $\boldsymbol{w}$ and introduce an additional map $\Psi_{N, \tau}$ to stand for the solution of

$$
\dot{\omega}_{r}=T_{\|}, \quad \dot{v}=\boldsymbol{v} \times \boldsymbol{T}^{*} .
$$

Remembering that $E$ was already attached to some of the Hamiltonian maps, we have $\dot{u}=0$. At this point we typically meet the situation in which $\boldsymbol{T}$ can be a fairly complicated function of $\boldsymbol{v}, \omega_{r}$, and $u$ but at the same time a very small vector when compared to $\{\boldsymbol{v}, \mathcal{K}\}$. Hoping that the latter advantage will pay for the former inconvenience, we can use the simple Euler-type approximation for $\omega_{r}$ and the so-called Lie-Euler approximation for $\boldsymbol{v}$ :

$$
\Psi_{N, \tau} \omega_{r}=\omega_{r}+\tau T_{\|}, \quad \Psi_{N, \tau} \boldsymbol{v}=\mathbf{K}\left(\boldsymbol{T}^{*}, \tau\right) \boldsymbol{v}
$$

where $\mathbf{K}\left(\boldsymbol{T}^{*}, \tau\right)=\exp \left[-\tau \mathbf{Q}\left(\boldsymbol{T}^{*}\right)\right]$ is a rotation matrix similar to $\mathbf{M}$ but with $T_{1}^{*}, T_{2}^{*}$, and $-T_{3}^{*} / 2$ instead of $\mathcal{A}, \mathcal{B}$, and $\mathcal{C}$, respectively. Thanks to the smallness of the rotation angle, it can be more convenient to approximate the matrix exponential by means of the Cayley transform (Iserles 2001):

$$
\begin{aligned}
\mathbf{K}\left(\boldsymbol{T}^{*}, \tau\right) & \approx \mathbf{K}_{c}\left(\boldsymbol{T}^{*}, \tau\right) \\
& =\left[\mathbf{1}-\frac{\tau}{2} \mathbf{Q}\left(\boldsymbol{T}^{*}\right)\right]\left[\mathbf{1}+\frac{\tau}{2} \mathbf{Q}\left(\boldsymbol{T}^{*}\right)\right]^{-1},
\end{aligned}
$$

where $\mathbf{1}$ is a $3 \times 3$ unit matrix; $\mathbf{K}_{c}$ is an orthogonal matrix, so it does not change the length of $\boldsymbol{v}$, and it differs from $\mathbf{K}$ by terms of order $(1 / 12)\left(T^{*} \tau\right)^{3}$. The explicit form of $\mathbf{K}_{c}\left(\boldsymbol{T}^{*}, \tau\right)$ is

$$
\mathbf{K}_{c}\left(\boldsymbol{T}^{*}, \tau\right)=1+\frac{2 \tau}{4+\tau^{2}\left(T^{*}\right)^{2}} \mathbf{k},
$$

where the elements of $\mathbf{k}$ are

$$
\begin{gathered}
k_{11}=-\tau\left[\left(T_{2}^{*}\right)^{2}+\left(T_{3}^{*}\right)^{2}\right], \quad k_{12}=2 T_{3}^{*}+\tau T_{1}^{*} T_{2}^{*}, \\
k_{13}=-2 T_{2}^{*}+\tau T_{1}^{*} T_{3}^{*}, \quad k_{21}=-2 T_{3}^{*}+\tau T_{1}^{*} T_{2}^{*}, \\
k_{22}=-\tau\left[\left(T_{1}^{*}\right)^{2}+\left(T_{3}^{*}\right)^{2}\right], \quad k_{23}=2 T_{1}^{*}+\tau T_{2}^{*} T_{3}^{*}, \\
k_{31}=2 T_{2}^{*}+\tau T_{1}^{*} T_{3}^{*}, \quad k_{32}=-2 T_{1}^{*}+\tau T_{2}^{*} T_{3}^{*}, \\
k_{33}=-\tau\left[\left(T_{1}^{*}\right)^{2}+\left(T_{2}^{*}\right)^{2}\right] .
\end{gathered}
$$

In some simple cases it may happen that at least some of equations (86) can be solved exactly. For example, the averaged tidal torque is a linear function of the spin rate (Gladman et al. 1996), allowing the exact solution for $\omega_{r}$. In such cases it is better to replace the Euler approximation with the actual solution, even at the expense of the computation time. We also noticed that if $\boldsymbol{T}$ depends on $\omega_{r}$, better results are obtained if the torque in equation (87) is evaluated at the midpoint $\frac{1}{2}\left(\omega_{r}+\Psi_{N, \tau} \omega_{r}\right)$.

Including a non-Hamiltonian torque in LP3 or LP2 results in the following leapfrog algorithms:

$$
\begin{aligned}
& \boldsymbol{w}(t+h)=\Psi_{N, h / 2} \circ \Psi_{0, h / 2} \circ \Psi_{1, h / 2} \circ \Psi_{2, h} \circ \Psi_{1, h / 2} \\
& \circ \Psi_{0, h / 2} \circ \Psi_{N, h / 2} \boldsymbol{w}(t)+O\left(h^{3}\right) \text {, } \\
& \boldsymbol{w}(t+h)=\Psi_{N, h / 2} \circ \Psi_{1, h / 2} \circ \Psi_{20, h} \\
& \circ \Psi_{1, h / 2} \circ \Psi_{N, h / 2} \boldsymbol{w}(t)+O\left(h^{3}\right) \text {, }
\end{aligned}
$$

respectively.

\section{NUMERICAL TESTS}

\subsection{Hamiltonian Test Cases}

Our first test case was adapted from studies of the rotational dynamics of the asteroid 433 Eros (Vokrouhlický et al. 2005). All parameters important in the Hamiltonian case are encapsulated in

$$
\alpha(t)=\alpha_{0}+\alpha_{1} \cos \left(\nu_{1} t+\alpha_{1,0}\right),
$$

where $\alpha_{0}=165^{\prime \prime} \mathrm{yr}^{-1}, \alpha_{1}=2^{\prime \prime} \mathrm{yr}^{-1}, \nu_{1}=10^{\prime \prime} \mathrm{yr}^{-1}$, and $\alpha_{1,0}=10^{\circ}$, and in

$$
q(t)+i p(t)=F_{1} \exp i\left(s_{1} t\right)+F_{2} \exp i\left(2 s_{1} t+f_{2}\right),
$$

with $F_{1}=\sin 7^{\circ} .5, s_{1}=-20^{\prime \prime} \mathrm{yr}^{-1}, F_{2}=\sin 1^{\circ}$, and $f_{2}=45^{\circ}$. We arbitrarily choose initial obliquity $\varepsilon=60^{\circ}$ and longitude $\lambda=45^{\circ}$. The resulting motion of the rotation pole proved to be quasi-periodic and hence well suited for the accuracy tests. The most prominent periods have an order of magnitude of about $10^{4}$ yr. Obliquity oscillates between $57^{\circ}$ and $76^{\circ}$, while longitude circulates through the full $360^{\circ}$ range.

LP2 and LP3 leapfrogs were compared with the reliable, general purpose integrator RA15 (Everhart 1985), an implicit collocation method of the 15th order with Gauss-Radau nodes and an automatic step-size adjustment. Our tests were performed over an interval of $1 \mathrm{Gyr}$, typical for long-term simulations. Pessimistic error estimates of the reference trajectory generated by RA15 were provided by back-and-forth integration, which took two runs of $12 \mathrm{hr}$ each on a $2 \mathrm{GHz}$ Pentium PC. The maximum forward-minus-backward differences reached 0.0007 in $\lambda$ and 0.00006 in $\varepsilon$.

We experimented with various step length values of LP2/LP3 leapfrogs: $0.1,0.25,0.5,1,2.5,5,10,25,50$, and $100 \mathrm{yr}$. The computation time was practically a linear function of $h^{-1}$, with benchmark values at $h=1 \mathrm{yr}$, i.e., $10^{9}$ steps, equal to $210 \mathrm{~s}$ for LP2 and $280 \mathrm{~s}$ for LP3. The results are summarized in Figures 1 and 2 .

In the absence of other integrals of motion, the conservation of the norm $v=1$ is the only internal quality test of the leapfrogs. We have recorded the maximum values attained by $|1-v|$ during the entire $1 \mathrm{Gyr}$ integration interval and plotted them for different step-size values. Figure 1 indicates that the error in $v$ is 


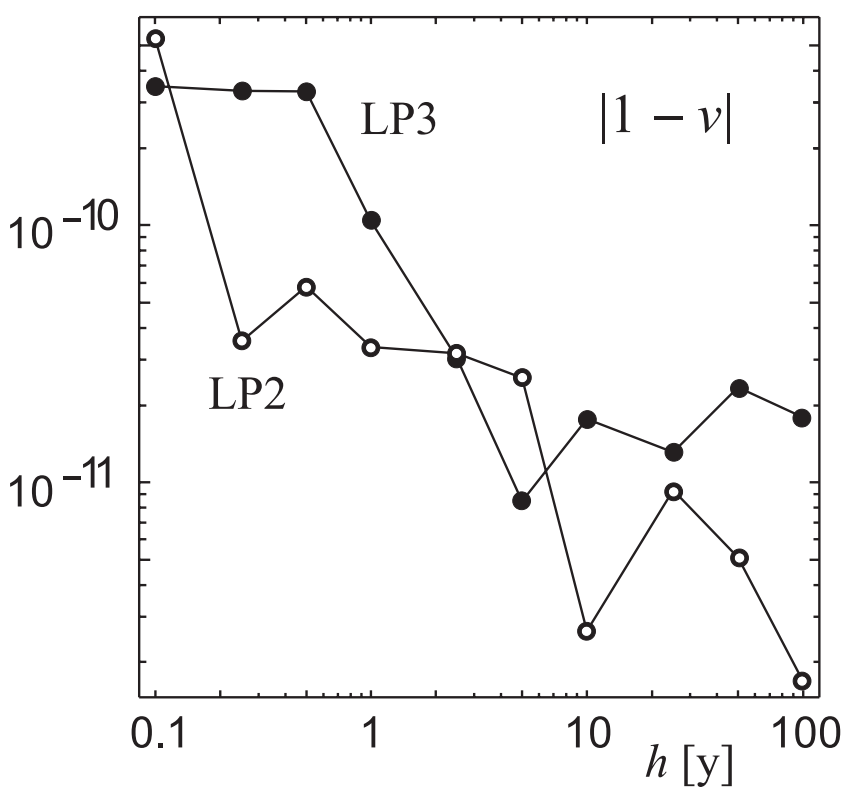

Fig. 1.-Orthogonality defect errors $|1-v|$ of LP2 (open circles) and LP3 ( filled circles) in the Hamiltonian case. Their dependence on $h$ suggests a roundoff origin.

entirely from round-off, increasing when $h$ is diminished. Interestingly, we found that RA15 conserves $v$ equally well (or better), on the level of $10^{-12}$.

Figure 2 shows the influence of step size $h$ on the maximum differences between the LP2/LP3 and RA15 solutions over a 1 Gyr interval. Almost perfect $\delta \propto h^{2}$ dependence is visible for $h$ between 5 and 50 yr. We recall that if the local truncation error of the method is $O\left(h^{3}\right)$, reducing the step size to $h / n$ requires $n$ times more steps; hence, the global truncation error scales as $h^{2}$. The curves bend on both ends. At higher $h$ it is the effect of approaching $360^{\circ}$ in $\delta \lambda$ and $19^{\circ}$ (the oscillations range)

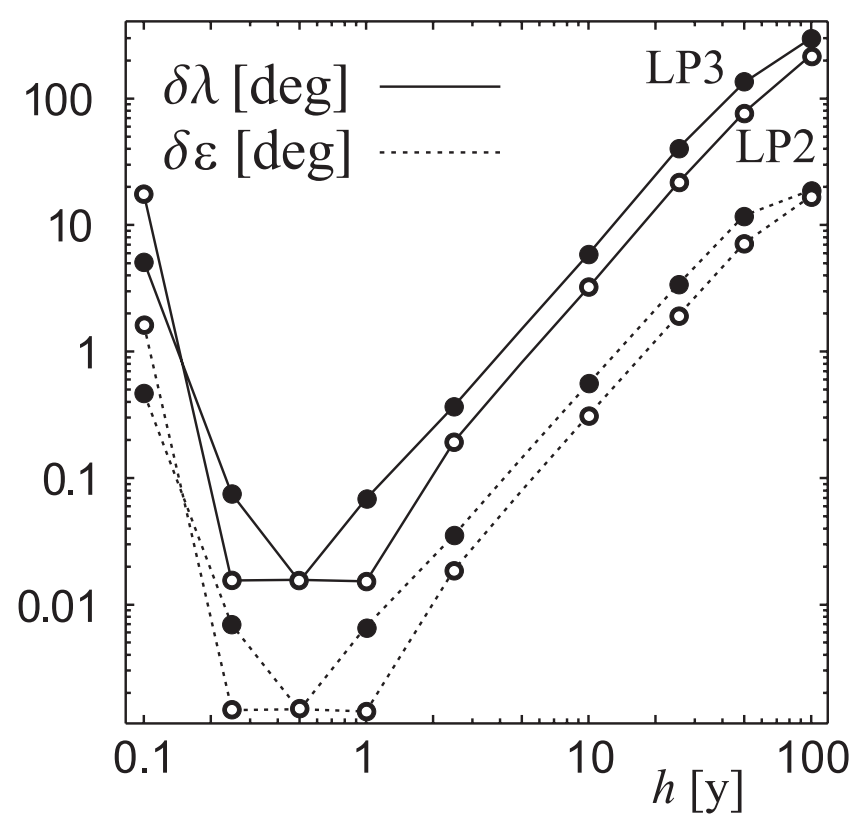

Fig. 2.-Absolute values of the differences between the RA15 results and LP2 (open circles) or LP3 ( filled circles) in longitude and obliquity for the Hamiltonian case. The optimum step size separating truncation and round-off errors is close to $h=0.5 \mathrm{yr}$.

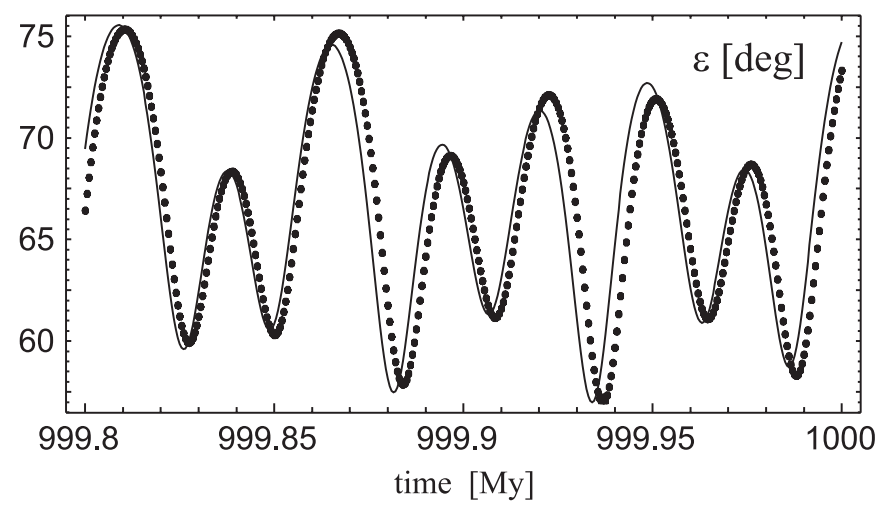

FIG. 3.-Obliquity during the last $0.2 \mathrm{Myr}$ of the $1 \mathrm{Gyr}$ interval in the Hamiltonian case. Circles refer to LP2 with $h=50 \mathrm{yr}$ and the solid line to RA15. The differences appear to be quasi-periodic, indicating the absence of spurious energy drift.

in $\delta \varepsilon$. In the small $h$ domain, the influence of round-off errors inverts the dependence of accuracy on step size. The best accuracy attained by LP2 was $\delta \lambda=0.015$ and $\delta \varepsilon=0.0014$ for $h=1 \mathrm{yr}$. LP3 attained a similar accuracy, but the optimum step size was shorter, namely, $h=0.5 \mathrm{yr}$.

Although in this example it proved possible to obtain an accuracy that could be considered fairly high for a second-order method, the usual applications of symplectic or Lie-Poisson methods do not aim at high-accuracy ephemeris generation. This kind of method serves to study qualitative properties of motion; hence, larger step lengths can be used thanks to the precious property that their errors affect oscillation amplitudes and frequencies only in a quasi-periodic manner. In order to demonstrate that this is the case for our Lie-Poisson integrators, Figure 3 shows $\varepsilon(t)$ computed from the results of the last $2 \times 10^{5} \mathrm{yr}$ integrated by LP2 with $h=50 \mathrm{yr}$ (points separated by $500 \mathrm{yr}$ ), together with the RA15 results (solid line). We can see that the main source of the $\delta \varepsilon$ differences plotted in Figure 2 is the phase shift of the oscillations. The maxima and minima of the oscillations look to be modulated with some spurious quasi-periodic terms not exceeding $1^{\circ}$, much less than the $7^{\circ}$ induced by the phase shift. Let it be recalled that in this case LP2 required only $10^{-4}$ of the RA15 CPU.

Equations (42) possess an additional integral of motion in the special case known as the Colombo top (Colombo 1966; Henrard \& Murigande 1987). When $\alpha=$ const and $q+i p$ consists of a single periodic term,

$$
q(t)+i p(t)=F_{1} \exp i\left(s_{1} t+f_{1}\right)
$$

the modified Hamiltonian

$$
\mathcal{H}_{\mathrm{C}}=\mathcal{H}^{\prime}+s_{1} z
$$

is constant along all trajectories. We checked the conservation of $\mathcal{H}_{\mathrm{C}}$ using the same initial conditions and parameters $\alpha_{0}$, $F_{1}$, and $s_{1}$ as in the previous case, with $f_{1}=0$. In accordance with theory, $\mathcal{H}_{\mathrm{C}}$-values computed by LP2 and LP3 oscillate around the constant mean value with amplitudes proportional to $h^{2}$. For $h \leq 10 \mathrm{yr}$ the round-off errors became visible, causing the secular trend in the relative error of $\mathcal{H}_{\mathrm{C}}$. For LP2 it was equal to $-7 \times 10^{-17} \mathrm{yr}^{-1}$ for $h=10 \mathrm{yr}$, and the total drift over 1 Gyr amounted to 0.15 of the oscillating component of the error. The secular trend was also visible in the results of LP3, 
but it was over 10 times smaller. In the case of the Colombo top, LP2 proved to be more vulnerable to round-off error accumulation.

\subsection{Non-Hamiltonian Torque Tests}

Choosing a good non-Hamiltonian torque for performance tests is a problematic task in an asteroidal type problem. Tidal torques can have a simple form, but their realistic magnitudes are too small for an asteroid to induce any significant phenomena. On the other hand, the Yarkovsky-O'Keefe-Radzievskii-Paddack (YORP) torques (Rubincam 2000; Capek \& Vokrouhlický 2004) are awkward in modeling, and readers might be unable to repeat our computations. As a compromise, we decided to use the averaged tidal torque model of Gladman et al. (1996),

$$
\boldsymbol{T}=-\frac{\gamma}{2} \boldsymbol{v}-\gamma\left(0,0, \frac{z}{2}-\frac{n}{\omega_{r}}\right)^{T}
$$

where $n$ is the orbital mean motion, but adopting a YORP-based value of $\gamma$. We added this torque to the Colombo top problem with the same parameters and initial conditions as in $\S 5.1$. Accordingly, the torque was time-independent with constant $n=$ 0.56 day $^{-1}$ and a constant parameter $\gamma$. The value of $\gamma$ was chosen such that $\omega_{r} \gamma=10^{-9} \mathrm{yr}^{-2}$, according to the order of magnitude of the YORP torque for 433 Eros computed by Čapek \& Vokrouhlický (2004). The initial value of the rotation rate, now explicitly required, was set to the Eros value of $\omega_{r}=$ $1640^{\circ}$ day $^{-1}$.

Like before, we first used the RA15 method to generate a reference trajectory over $1 \mathrm{Gyr}$, with the backward integration providing the accuracy estimates: 0.0002 in $\lambda, 0^{\circ} .00001$ in $\varepsilon$, and $4 \times 10^{-12}$ for the relative (i.e., divided by the initial value of $\omega_{r}$ ) error of the rotation rate. By the end of the integration interval, $\omega_{r}$ decreased to almost half its initial value, reaching $935^{\circ}$ day $^{-1}$. At the same time, the mean obliquity increased

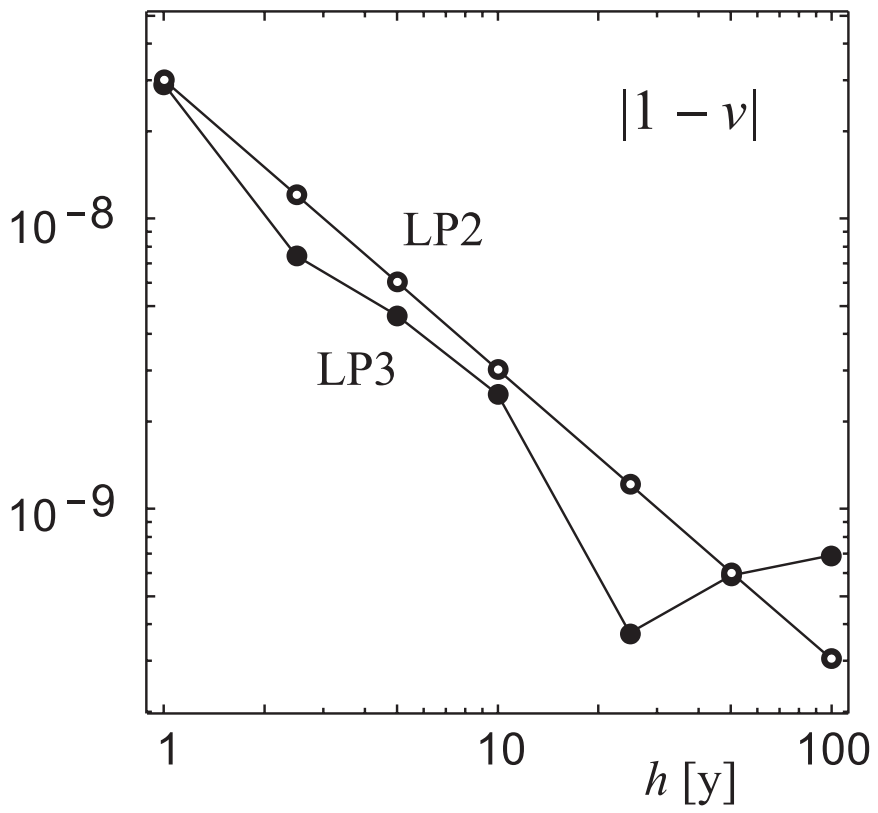

FIG. 4.-Orthogonality defect errors $|1-v|$ of LP2 (open circles) and LP3 ( filled circles) in the non-Hamiltonian case. Their values are higher than in the Hamiltonian case, and LP2 proves more vulnerable to round-off errors than LP3.

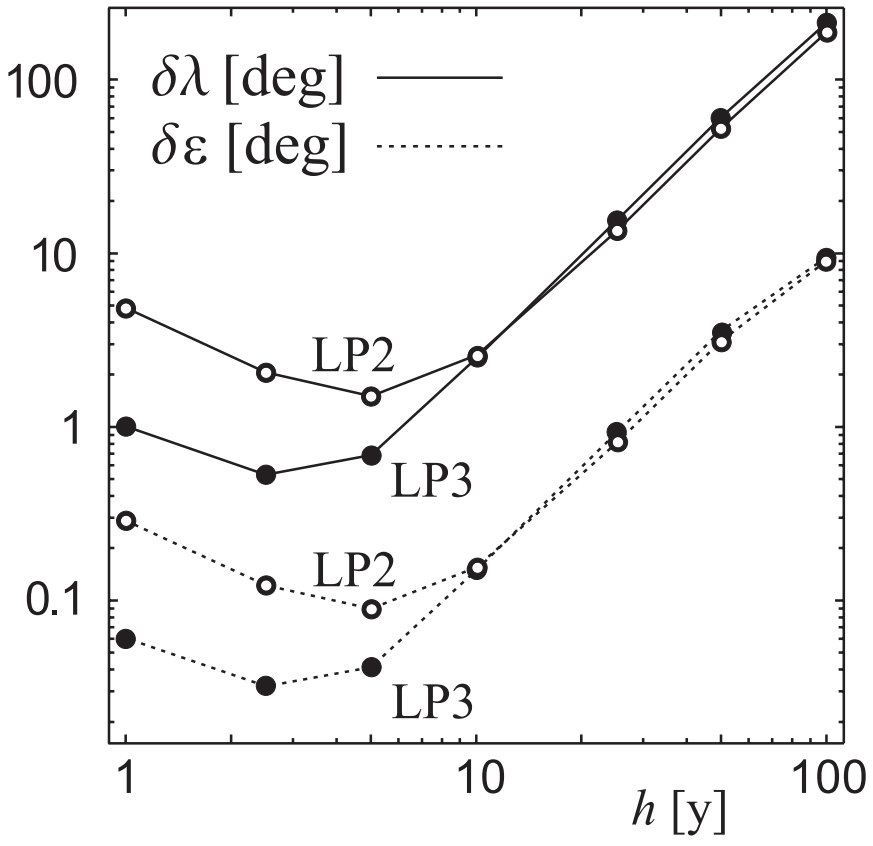

FIG. 5.-Absolute values of the differences between the RA15 results and LP2 (open circles) or LP3 (filled circles) in longitude and obliquity in the non-Hamiltonian case. Round-off errors dominate for $h<5 \mathrm{yr}$.

from $65^{\circ}$ to $74^{\circ}$, whereas the amplitude of oscillations around the mean $\varepsilon$ slightly decreased from 8.5 to $7^{\circ}$.

We tested seven different values of $h$ for the non-Hamiltonian LP2 and LP3 leapfrogs: 1, 2.5, 5, 10, 25, 50, and 100 yr. Figures 4-6 summarize the results. The best accuracy attained is worse than in the Hamiltonian case. This is mostly due to the more significant influence of the round-off errors. Another important point is related to the dependence of the local truncation on the rotation rate $\omega_{r}$. The value of $h$ is fixed in the course of the integration, but the smaller $\omega_{r}$ becomes, the more the local truncation error grows. Of course, selecting the integration step

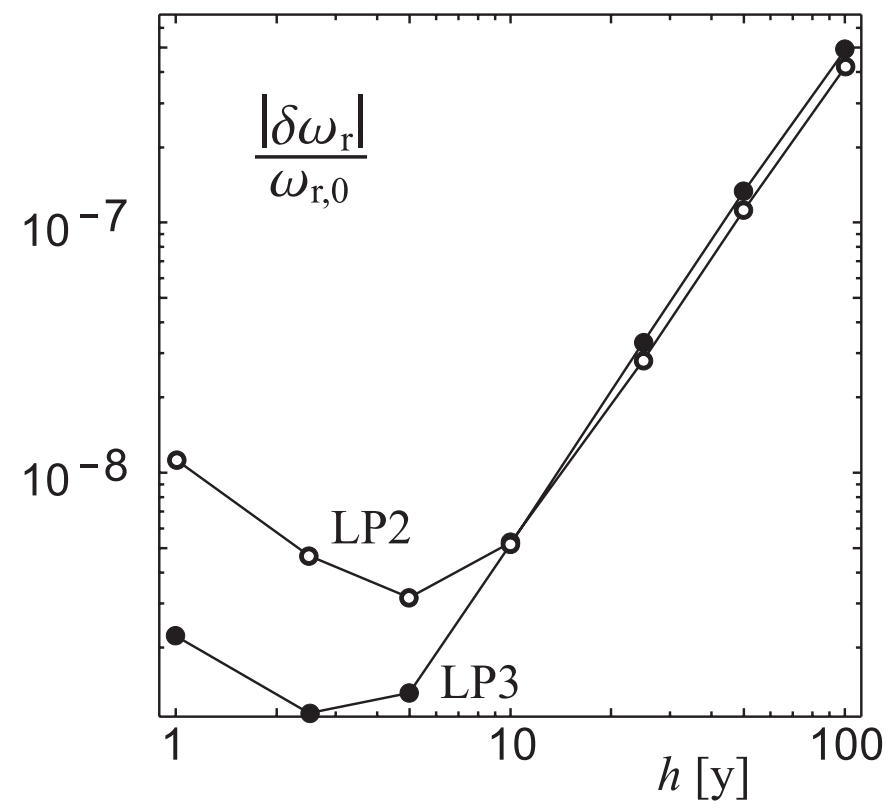

Fig. 6.-Absolute values of relative errors in rotation frequency with respect to the RA15 results. 
according to the expected final value of $\omega_{r}$ increases the roundoff error accumulation.

\section{CONCLUSIONS}

The Lie-Poisson formalism is an extension of the symplectic approach that leads to a simple and elegant treatment of the rotation problem. Most of the transformations and derivations presented in $\S 2$ can be cut short if more elements of the Lie groups and Lie algebra are introduced, but we did not want to repel practically oriented readers who are less familiar with the theoretical background of geometric integrators.

The two integration methods that we have presented and compared are somehow complementary: LP2 performs better (both faster and more accurately) than LP3, but it is more vulnerable to the round-off error accumulation due to the back-and-forth rotation (eq. [68]) in the special case of the Colombo top. Both methods proved very efficient, providing reliable results within a significantly shorter computation time with respect to general purpose integrators. Adding the non-Hamiltonian torque slightly degrades the performance of both methods, but this should be expected because of the Euler or Lie-Euler type approximation that was used instead of the exact solution required by the splitting type algorithms. We can hint, however, that once the actual form of the torque is known, a better solution or approximation to equations (82) and (83) can be sought. We have already begun the studies on the particular case of the YORP torque, to be reported in a future paper.

The research was supported by Polish State Committee of Scientific Research grant 1 P03D 02027 (S. B.) and grant 205/05/2737 of the Grant Agency of the Czech Republic (D. V.), as well as NSF grant AST 00-74163 and NASA's Planetary Geology and Geophysics program, grant NAG 513038 (D. N.).
Atobe, K., Ida, S., \& Ito, T. 2004, Icarus, 168, 223

Battin, R. H. 1987, An Introduction to the Mathematics and Methods of Astrodynamics (New York: AIAA)

Bertotti, B., Farinella, P., \& Vokrouhlický, D. 2003, Physics of the Solar System (Dordrecht: Kluwer)

Budd, C., \& Piggott, M. 2003, in Handbook of Numerical Analysis, Vol. 11, ed. P. Ciarlet \& F. Cucker (Amsterdam: North Holland), 35

Capek, D., \& Vokrouhlický, D. 2004, Icarus, 172, 526

Colombo, G. 1966, AJ, 71, 891

Correia, A. C. M., \& Laskar, J. 2001, Nature, 411, 767 2003, Icarus, 163, 24

Correia, A. C. M., Laskar, J., \& Néron de Surgy, O. 2003, Icarus, 163, 1

Dobrovolskis, A. R. 1980, Icarus, 41, 18

Dobrovolskis, A. R., \& Ingersoll, A. P. 1980, Icarus, 41, 1

Everhart, E. 1985, in IAU Colloq. 83, Dynamics of Comets, ed. A. Carusi \& G. B. Valsecchi (Dordrecht: Reidel), 185

Feynman, R. 1967, The Character of Physical Law (Cambridge: MIT Press)

Gladman, B., Quinn, D. D., Nicholson, P., \& Rand, R. 1996, Icarus, 122, 166

Goldreich, P., \& Peale, S. J. 1970, AJ, 75, 273

Hairer, E. 2001, BIT, 41, 996

Hairer, E., Lubich, C., \& Wanner, G. 2003, Acta Num., 12, 399

Hamilton, D. P., \& Ward, W. R. 2004, AJ, 128, 2510

Henrard, J., \& Murigande, C. 1987, Celest. Mech., 40, 345

Iserles, A. 2001, Found. Comput. Math., 1, 129

Kaasalainen, M. 2004, A\&A, 422, L39

Laskar, J. 1986, A\&A, 157, 59

Laskar, J., Correia, A. C. M., Gastineau, M., Joutel, F., Levrard, B., \& Robutel, P. 2004a, Icarus, 170, 343

Laskar, J., Levrard, B., \& Mustard, J. F. 2002, Nature, 419, 375

Laskar, J., \& Robutel, P. 1993, Nature, 361, 608

2001, Celest. Mech. Dyn. Astron., 80, 39

Laskar, J., Robutel, P., Joutel, F., Gastineau, M., Correia, A. C. M., \& Levrard, B. 2004b, A\&A, 428, 261

\section{REFERENCES}

McLachlan, R. I. 1996, Fields Inst. Commun., 10, 141

McLachlan, R. I., \& Quispel, G. R. W. 2002, Acta Num., 11, 341

Néron de Surgy, O., \& Laskar, J. 1997, A\&A, 318, 975

Newcomb, S. 1895, Astron. Papers, 6, 2

Olver, P. J. 1993, in Graduate Texts in Mathematics, Vol. 107, ed. S. Axler,

F. W. Gehring, \& K. A. Ribet (New York: Springer), 378

Peale, S. J. 1969 , AJ, 74, 483 . 1974, AJ, 79, 722

Rochester, M. G. 1976, Geophys. J. RAS, 46, 109

Rubincam, D. P. 2000, Icarus, 148, 2

Rubincam, D. P., Rowlands, D. D., \& Ray, R. D. 2002, J. Geophys. Res., 107, 3-1

Sheng, Q. 1989, IMA J. Num. Anal., 9, 199

Skoglöv, E. 1997, Planet. Space Sci., 45, 439

1998, Planet. Space Sci., 47, 11

Slivan, S. M. 2002, Nature, 419, 49

Touma, J., \& Wisdom, J. 1994a, AJ, 107, 1189 1994b, AJ, 108, 1943

Vokrouhlický, D., Bottke, W. F., \& Nesvorný, D. 2005, Icarus, 175, 419

Vokrouhlický, D., \& Čapek, D. 2002, Icarus, 159, 449

Vokrouhlický, D., Nesvorný, D., \& Bottke, W. F. 2003, Nature, 425, 147

Ward, W. R. 1973, Science, 181, 260 . 1974, J. Geophys. Res., 79, 3375

1975, Science, 189, 377

1979, J. Geophys. Res., 84, 237 1980, Icarus, 50, 444

Ward, W. R., \& de Campli, W. M. 1979, ApJ, 230, L117

Ward, W. R., \& Hamilton, D. P. 2004, AJ, 128, 2501

Whittaker, E. T. 1944, A Treatise on the Analytical Dynamics of Particles and Rigid Bodies (New York: Dover)

Wisdom, J., Holman, M., \& Touma, J. 1996, Fields Inst. Commun., 10, 217 